\title{
Expert Opinion Paper
}

\section{Human Musculoskeletal Models of Disuse}

\author{
Yannis Dionyssiotis ${ }^{1}$, Athina Kapsokoulou², Eleni Samlidi ${ }^{3}$, Georgia latridou $^{4}$ \\ ${ }^{1}$ Physical Medicine \& Rehabilitation Department, European Interbalkan Medical Center, Thessaloniki, Greece \\ ${ }^{2}$ Medical School of Patras, Rio, Patras, Greece \\ ${ }^{3}$ Medical School of Thessaly, Larissa, Greece \\ ${ }^{4}$ Physical Therapy Unit "Physiospot", Athens, Greece
}

All published work is licensed under Creative Common License CC BY-NC-SA 4.O (Attribution-NonCommercial-ShareAlike)

Keywords: Disuse, Mechanostat, Muscle-bone interaction, Bed-rest studies, Spinal cord injury

\section{The development of Frost's mechanostat theory}

According to Frost's mechanostat theory when the bone receives strains of about 800 to 1600 micro-strain retains its strength; increased strains promote formation (remodeling) up to a fracture point, and when bone is a low loading status receiving strains below 800 micro-strains starts remodeling moves to the opposite direction of resorption ${ }^{1,2}$. The picture (Figure 1) shows the substantially variable bone response dependent upon the degree of deformation (strain) ${ }^{3}$. The theory is developed in the "musculoskeletal unit" with the employment of real bone mineral density measurement technology i.e. peripheral quantitative computed tomography, pQCT (Figure 2). An increase in muscle area (i.e. muscle) is not positively correlated with trabecular bone mineral density, but this is not the case with the bone mineral content which is increased. Increased bone mineral content is leading to increased bone strength ${ }^{4}$.

The final development of this theory was called "Utah Paradigm of Skeletal Physiology": Exercise causes an increase in bone mass and strength more in children than adults, and is marginally effective in increasing bone mass in the elderly. The reduced effectiveness of exercise in the frame of the elderly can be attributed to decreased sensitivity of this aging skeleton to mechanical stimuli. One way to overcome this poor response to the aged frame could be the use of promising non-mechanical factors to overcome the decreased anabolic sensitivity and increase bone mass and strength. We already know that estrogen modify the set points of the mechanostat. As long as estrogens, for example in a woman, are within a normal range, common loading (daily activities) maintains a relatively stable state in the bone. Estrogen deficiency in menopause, therefore, results in a situation that a common load cannot maintain the bone any more. Bone receives a disuse signal because the set point of the mechanostat is moving towards resorption direction in the presence of loading strains of the former common loading activities and the absence of the prophylactic action of estrogen. Similar modification of the set point can be done with other interventions such as anabolic drugs i.e. teriparatide towards formation (remodeling with positive formation/ resorption balance) $)^{5}$.

\section{Muscle-bone interactions}

A simple reduction of physiological activity levels represents the first element with respect to disuse. More dramatic changes occur with prolonged bed rest or weightlessness in space, especially in the bones. These conditions have the effect of reducing the compressive forces typically exerted on bones standing up, in normal gravity conditions. Moreover, since the ground reaction forces are removed in space, the muscle strength needed to promote movement is significant reduced. The architecture of the cortical and trabecular bone in the axial and peripheral skeleton is adapted according to the mechanical loading of the muscle contraction and gravity. Muscle and bone tissue is starting to reduce the adaptation to the load during bed rest within days. The main elements of bed immobilization that contribute to changes in the musculoskeletal system are: (a) the lack of normal load exerted on the bones in the upright posture, and $(b)$ the reduction in the number

Corresponding author: Yannis Dionyssiotis, MD, PhD, Physical Medicine \& Rehabilitation Department, European Interbalkan Medical Center, 10 Asclepiou Str., Pylaia Thessaloniki, 57001 , Greece

E-mail: yannis_dionyssiotis@hotmail.com

Edited by: George P. Lyritis

Accepted 29 March 2016 

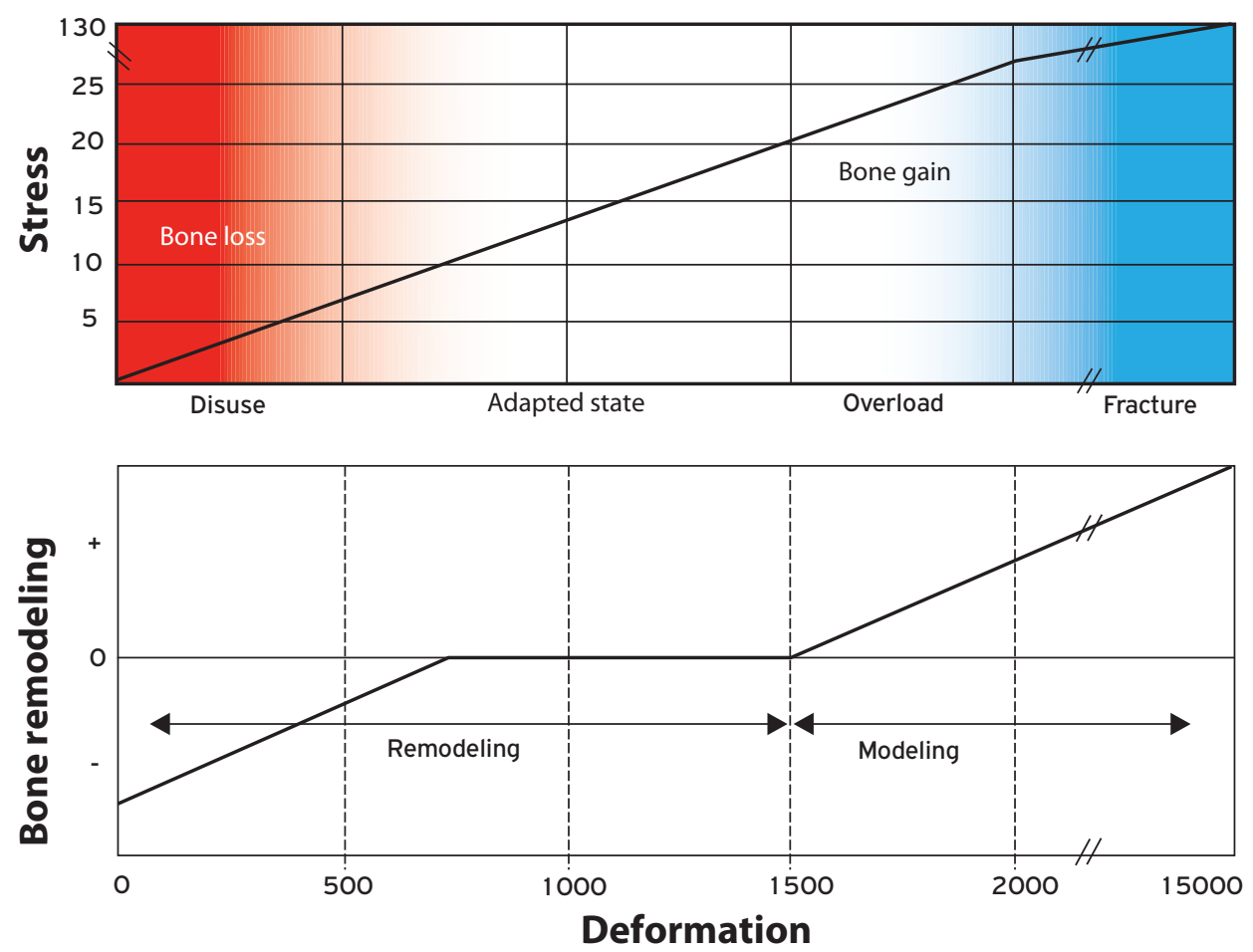

Figure 1. The variability of deformation in bone depends on the degree of loading.

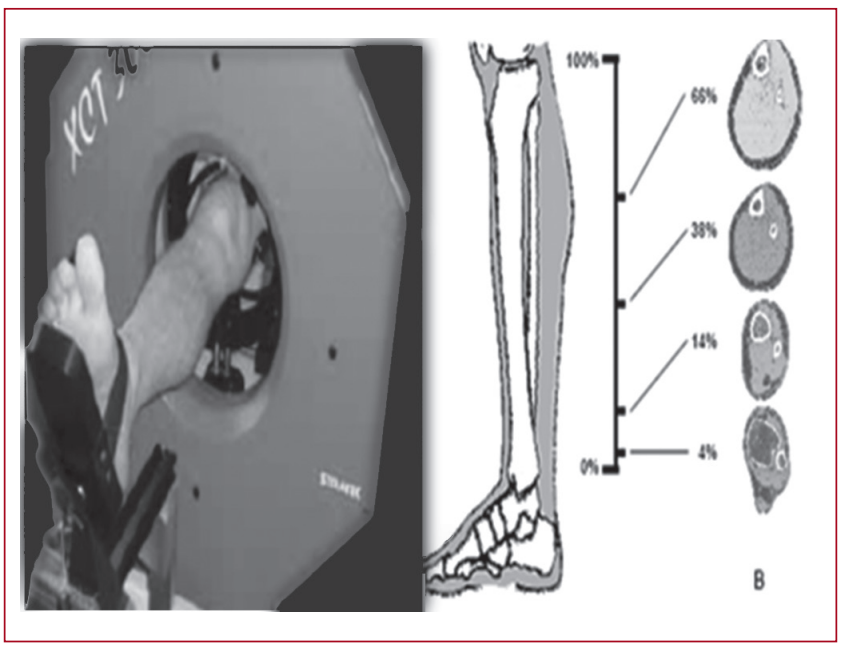

Figure 2. Measurement of bone parameters using peripheral quantitative computed tomography ( $\mathrm{Q}$ QCT) XCT 3000 Stratec Medizintechnik (Pforzheim, Germany) in the tibia.

and intensity of muscle contractions, particularly in the muscles of posture ${ }^{6}$.

Muscles have been identified as the main source of anabolic stimuli to the bone, but the exact nature of the stimuli which induce bone formation has not been fully clarified. On the other side an opposite action results through the action of a disused muscle to bone ${ }^{7}$. The positive effects of muscle and high intensity strength training in postmenopausal women have been already published in meta-analysis studies ${ }^{8,9}$. In the recent guidelines published from Hellenic Institution of Osteoporosis in 2013 was stated that there are studies that show the positive effects of high-intensity exercise in the hip and spine with a high degree of reliability and this is in line with studies employed sophisticated computed tomography ${ }^{10}$. The effect of exercise is moderate and region specific and mainly affects the cortical rather than the trabecular bone ${ }^{11,12}$.

However, there is another form of this relationship: the disuse status. Immobilization studies in bed organized by a team of pioneer scientists in the field using specific methodology with the head down. During these studies subjects were lying in bed for 89 days and then were mobilized. It was observed that muscle loss started immediately from the first day. The loss of bone which was counted with very specialized technology began after 30 days. They also noted that in the $89^{\text {th }}$ day when the experiment ended and people got up the muscles immediately began to regain size, while on the contrary bone continued to get lost. Rittweger and Felsenberg revealed a reduction of the mineral of tibial bone during immobilization in bed (Toulouse bed rest study). This loss was reduced by exercise and drug treatment with pamidronate. The loss of bone mineral in people who received pamidronate 
and those carried out resistance training suggests that these countermeasures were not fully effective for the treatment of immobilization in bed. However, the observation that loss of bone mineral in some individuals of the same study control group was very slow is indicating of intervention of factors other than mechanical use. Such other factors could be local (i.e. recruitment of osteoclasts from bone marrow) or systemic (i.e. renal calcium excretion ability). The rate of the structural bone changes beyond those observed in the muscles during bed immobilization or unloading may be in some extent due to slow remodelling of bone tissue. Nevertheless, there is a strong systematic guidance in bone loss, as shown by the strong symmetry changes in bone mineral content (BMC) at the left and right tibia. Another set of observations could be summed up as a result of "lag". The loss of bone mineral really begins only after four weeks' immobilization and continued for at least two weeks after the resumption of walking. The latter finding might be due to an increase of micro-damages, which led to the remodelling of the activity, evidenced by the loss of bone mass (i.e. BMC) in the metaphyseal bone region which was negligible during immobilization in bed but substantial during the resumption of walking. However, the late onset of bone loss is difficult to be explained by osteoclasts' activation time (on the order of days). Even more puzzling is the rapid increase in the BMC in the diaphysis of the tibia during the resumption of gait ${ }^{13}$.

Strict bed rest, four months' duration, in healthy male volunteers, aged 19-52 years, resulted in significant decreases in bone mineral density of the spine $(-4 \%)$, femoral neck (-4\%) and tibia (-2\%), and most obvious was the loss of the heel $(10 \%)$. However, the BMD of the radius showed no change ${ }^{14}$. This finding reflects the comparable large reductions of the bone mineral content of the heel and the small reduction in the upper limbs during the non-loading condition of the body in space flight. The bones of the lower limb are exposed to frequent, long compressive loadings of $1 \mathrm{G}$ and experiencing greater reduction in daily loading activities from immobilization or conditions without gravity. Conversely, the use of the upper limbs increases during flight in space because it helps to movements and carrying out activities. Bone loss is usually observed mostly in loaded bones such as the long bones of the leg, the heel etc ${ }^{15}$. The mineral density of the vertebrae decreases by about $1 \%$ per week during immobilization in bed about 50 times higher than anticipated under normal bone loss conditions. Another study showed $25-40 \%$ loss in bone mass of the calcaneus, after 30-36 weeks of bed rest. The bones which are not loaded such as the radius and ulna, lose significantly less bone mass and, in some studies the skull actually showed an increase in mineral density (perhaps because of an extra new load in the supine position $)^{16}$.

In humans there are a sufficient number of muscle diseases which demonstrate that normal muscle function is essential for maintaining the skeleton. There are neurological diseases such as myasthenia Gravis, which is an autoimmune disease that destroys signals of acetylcholine at the neuromuscular junction. These patients experience a dramatic loss of muscle strength with secondary osteoporosis ${ }^{17}$. Further there is Duchenne muscular dystrophy, a disease in which the lack of dystrophin leads to muscle degeneration and long these patients suffer from alterations in geometrical characteristics of bone, and increased risk of fracture ${ }^{18}$. But there are other diseases in which a muscle disorder coexists with bone disease such as osteomalacia i.e. an insufficient mineralization of the bone and osteomalacia myopathy a proximal myopathy, particular in trunk, reported to be present in $60-75 \%$ of patients with vitamin D deficiency which is reversible ${ }^{19}$. The extreme example of disuse is the spinal cord injury. In a spinal cord injury there is a loss of muscle contractions due to the damage of the spinal cord. However, there are still some contractions either as muscle spasms or as spinal reflexes. One could argue that because there is such intense and permanent neurological damage in the spinal cord, the result of the great destruction of the bone is not because of muscle damage, but due a central mechanism. Several studies steer in this direction and emphasize that there is an involvement of the sympathetic nervous system (SNS). Such nervous influence is probably best understood for the sympathetic nervous system, which is thought to hamper bone formation and stimulate bone resorption. However, sympathetic nerve activity is decreased after $\mathrm{SCl}$, and, accordingly one should expect increases in bone mass and strength via this pathway ${ }^{20}$. From neurological point of view this idea is wrong. On the contrary, in all spinal cord injuries, even in low neurological level spinal cord subjects, there is a major muscle-bone loss. Indeed, in spinal cord injured subjects with SNS damage (i.e. with a high spinal cord lesion) bone and muscle mass is extremely reduced. Therefore, we expect that the higher the level of lesion the more deteriorated the image of the bone-muscle unit would be. However, this is not the case in spinal cord bone-muscle pathology. Actually bone-muscle unit depends on the degree of the lesion i.e. complete vs. incomplete (complete: an absence of sensory or motor function below the neurological level, including the lowest sacral segment and incomplete: partial preservation of motor and/or sensory function below the neurological level, including the lowest sacral segment). Patients with complete injuries have greater bone loss than those with an incomplete injury, as has already been shown in Brown-Sequard subjects (incomplete SCI), where BMD of the more paretic knee was lower than that of the stronger knee. In conclusion the key factor is clearly the relationship muscle and bone, i.e. how much residual muscle function is left to produce a specific loading-deformation to the bone ${ }^{21}$.

What exactly is happening in spinal cord injured subjects is the rapid loss and muscle atrophy that reaches an equilibrium at 6 months which is maintained mainly by reflexes of the upper motor neuron. Now days we are also aware of the myopathy changes this muscle is suffering: The disuse and lesion combined with spasticity, which occurs, affecting the nervous system and cause mutation of muscle fiber to type II, while there are other factors such as prevascular lesions, histochemical and pathological alterations acting synergistically in the muscles. This is not the case in sarcopenia where most muscle fibers type II are lost or transformed in type I. Type II muscle fibers degenerate, its 
diameter gets smaller and in addition through the impact of other inflammatory factors a different distribution of muscle fibers exists ${ }^{22}$. Recently some studies published negative results in mice's bones with the use of botulinum toxin (i.e. BOTOX $^{\circledR}$ ), which is an extremely powerful agent to block neuromuscular transmission. They found decreases in bone strength and mass in mice ${ }^{23}$. Basically the studies suggested that BOTOX $^{\circledR}$ had a deleterious effect in animal bones' especially trabecular bone ${ }^{24}$. However, in clinical practice in rehabilitation medicine all commercially available types of botulinum toxin (i.e. BOTOX ${ }^{\circledR} /$ DYSPORT $^{\circledR} /$ XEOMIN $^{\circledR}$ ) are commonly used in the treatment of focal spasticity and more research is needed in the future to clarify how we should proceed with these definitely useful drugs in patients with neurological damage and bone loss.

\section{References}

1. Frost HM. Bone "mass" and the "mechanostat": a proposal. Anat Rec 1987;219:1-9.

2. Frost HM. The mechanostat: a proposed pathogenic mechanism of osteoporoses and the bone mass effects of mechanical and nonmechanical agents. Bone Miner 1987;2:73-85.

3. Dionyssiotis Y. Exercise in Osteoporosis and Falls Prevention. Athens: Monography (in Greek) published for Hellenic Institution of Osteoporosis (HELIOS) Hylonome Editions; 2008.

4. Runge M, Schacht E. Multifactorial pathogenesis of falls as a basis for multifactorial interventions. J Musculoskelet Neuronal Interact 2005; 5: 127-34.

5. Jee WS, Tian XY. The benefit of combining non-mechanical agents with mechanical loading: a perspective based on the Utah Paradigm of Skeletal Physiology. J Musculoskelet Neuronal Interact 2005;5(2): $110-8$.

6. Bloomfield SA. Changes in musculoskeletal structure and function with prolonged bed rest. Med Sci Sports Exerc 1997;29(2): 197-206.

7. Bonaiuti D, Shea B, lovine R, et al. Exercise for preventing and treating osteoporosis in postmenopausal women. Cochrane Database Syst Rev 2002:3:CDO00333.

8. Martyn-St James M, Carroll S. High-intensity resistance training and postmenopausal bone loss: a meta-analysis. Osteoporos Int 2006; 17:1225-40.

9. Martyn-St James M, Carroll S. Meta-analysis of walking for preservation of bone mineral density in postmenopausal women. Bone 2008;43:521-31.

10. Hellenic Osteoporosis Foundation (HELIOS) Guidelines for the Diagnosis and Treatment of Osteoporosis in Greece. Athens, Greece: Hylonome Editions; 2009.

11. Hamilton CJ, Swan VJ, Jamal SA. The effects of exercise and physical activity participation on bone mass and geometry in postmenopausal women: a systematic review of PQCT studies. Osteoporos Int 2010; 21:11-23.

12. Polidoulis I, Beyene J, Cheung AM. The effect of exercise on PQCT parameters of bone structure and strength in postmenopausal women - a systematic review and meta-analysis of randomized controlled trials. Osteoporos Int 2012;23:39-51.

13. Rittweger J, Felsenberg D. Patterns of bone loss in bed-ridden healthy young male subjects: results from the Long Term Bed Rest Study in Toulouse. J Musculoskelet Neuronal Interact 2003;3(4):290-1; discussion 2-4.

14. LeBlanc AD, Schneider VS, Evans HJ, et al. Regional changes in muscle mass following 17 weeks of bed rest. J Appl Physiol 1992; 73(5):2172-8.

15. Dudley GA, Duvoisin MR, Convertino VA, Buchanan P. Alterations of the in vivo torque-velocity relationship of human skeletal muscle following 30 days exposure to simulated microgravity. Aviat Space Environ Med 1989;60(7):659-63.

16. Grabois M, ed. Physical medicine and rehabilitation: the complete approach. Malden, Mass.; Oxford: Blackwell Science; 2000.

17. Yeh $\mathrm{JH}$, Chen $\mathrm{HJ}$, Chen $\mathrm{YK}$, Chiu HC, Kao CH. Increased risk of osteoporosis in patients with myasthenia gravis: a population-based cohort study. Neurology 2014;83(12):1075-9.

18. Houston C, Mathews K, Shibli-Rahhal A. Bone density and alendronate effects in Duchenne muscular dystrophy patients. Muscle Nerve 2014;49(4):506-11.

19. Bhan $A$, Rao AD, Rao DS. Osteomalacia as a result of vitamin $D$ deficiency. Endocrinol Metab Clin North Am 20 10;39(2):32 1-31.

20. Rittweger J, Gerrits K, Altenburg T, Reeves N, Maganaris CN, de Haan A. Bone adaptation to altered loading after spinal cord injury: a study of bone and muscle strength. J Musculoskelet Neuronal Interact 2006;6(3):269-76

21. Dionyssiotis Y. Spinal cord injury-related bone impairment and fractures: an update on epidemiology and physiopathological mechanisms. J Musculoskelet Neuronal Interact 2011;11(3):25765.

22. Dionyssiotis Y (2012). Body Composition in Spinal Cord InjuredParaplegic Men. In: Handbook of Anthropometry. Edited by: Preedy VR 2317-2339 Springer US ISBN: 978-1-4419-1788-1.

23. Warden SJ, Galley MR, Richard JS, George LA, Dirks RC, Guildenbecher EA, Judd AM, Robling AG, Fuchs RK. Reduced gravitational loading does not account for the skeletal effect of botulinum toxin-induced muscle inhibition suggesting a direct effect of muscle on bone. Bone 2013:54(1):98-105.

24. Marchand-Libouban H, Le Drévo MA, Chappard D. Disuse induced by botulinum toxin affects the bone marrow expression profile of bone genes leading to a rapid bone loss. J Musculoskelet Neuronal Interact 2013;13(1):27-36. 